\title{
Coordination of Fluoro Ligands Toward Sodium Ions Makes the Difference: Aqua Sodium Ions Act as a Brønsted Acid in Polymerization of Vinyl ethers and Styrenes.
}

\author{
Chi-Tsing Chang, Chuan-Lin Chen, Yi-Hong Liu, Shie-Ming Peng, Pi-Tai Chou* and
} Shiuh-Tzung Liu*

Department of Chemistry, National Taiwan University, Taipei, Taiwan 106

\section{Supporting Materials}

General information. All reactions were performed under a dry nitrogen atmosphere. Dichloromethane was dried over $\mathrm{CaH}_{2}$ and distilled under nitrogen. Nuclear magnetic resonance spectra were recorded in $\mathrm{CDCl}_{3}$ on either a Bruker AC-E 200 or AM-300 spectrometer.Gel permeation chromatography (GPC) data were obtained from a Waters Model 590 liquid chromatograph installed with a Lab Allience RI 2000 detector using THF as eluant at room temperature and polystyrene calibration curve for analyses.

Polymerization of vinyl ether. To a flask was placed with the aqua sodium salt, which was evacuated and flashed with nitrogen three times. Vinyl ether and dichloromethane were syringed into the flask under nitrogen atmosphere. The resulting mixture was stirred at room temperature for certain period. After the reaction was completed, the reaction mixture was concentrated. The residue was filtrated through silica gel column with elution of chloroform. The desired polymer was obtained upon concentration.

Poly(2,3-dihydrofuran) $)^{1}{ }^{1} \mathrm{H}$ NMR $\delta 3.72-3.52$ (br, $3 \mathrm{H}, \mathrm{OCH}-$ and $\left.\mathrm{OCH}_{2}-\right), 2.33$ 1.85 (br, $3 \mathrm{H}$, - $\mathrm{CH}-$ and $-\mathrm{CH}_{2^{-}}$).

Poly(i-butyl vinyl ether) ${ }^{2}:{ }^{1} \mathrm{H}$ NMR $\delta 3.43-3.36$ (br, $3 \mathrm{H}, \mathrm{O}-\mathrm{CH}-$ and $\left.\mathrm{O}-\mathrm{CH}_{2}-\right), 2.23$ 1.63 (br, $3 \mathrm{H},-\mathrm{CH}_{2}$ - and $\mathrm{CH}_{3} \mathrm{CH}-$ ), $0.90-0.82$ (br, $6 \mathrm{H},-\mathrm{CH}_{3}$ ).

Poly(p-methoxystyrene) $)^{3}:{ }^{1} \mathrm{H}$ NMR $\delta$ 7.2-6.3 (Br, 4H, Ar- $H$ ), 3.7- 3.9 (br, 3H, -O-CH3), 2.2- 1.25 (br, $3 \mathrm{H},-\mathrm{CH}_{2}-\mathrm{CH}$ ), 1.13 (terimal- $\mathrm{CH}_{3}$ ).

Poly(allyl vinyl ether) ${ }^{4}:{ }^{1} \mathrm{H}$ NMR $\delta 5.80-5.53(\mathrm{br}, 1 \mathrm{H},=\mathrm{CH}-), 5.10-5.30(\mathrm{br}, 2 \mathrm{H}$, $\left.=\mathrm{CH}_{2}\right), 4.0-4.2\left(\mathrm{br}, 2 \mathrm{H}, \mathrm{OCH}_{2^{-}}\right), 3.36-3.18(\mathrm{br}, 1 \mathrm{H}, \mathrm{O}-\mathrm{CH}-), 1.65-1.52(\mathrm{br}, 1 \mathrm{H}$, 
$-\mathrm{CH}-\mathbf{-}$.

The theoretical approach on vibration frequencies was performed by adopting UB3LYP method with the $3-21 \mathrm{G}^{*}$ basis set based upon the $\mathrm{x}$-ray structure. The charge on $\mathrm{F}$ is chosen to be either -0.1875 according to the natural population analysis (NPA) or -0.3402 via the electrostatic potential surface fitting according to ChelpG scheme. All calculations were done with Gaussian $03 .^{5}$

\section{References}

1. Chen, Y.-C.; Reddy, K. R.; Liu, S.-T. J. Organomet. Chem. 2002,656, 198

2. Lang, W.; Sarker, P. K.; Rimmer, S. Macromol. Chem. Phys. 2004, 205, 1011.

3. Cauvin, S.; Ganachaud, F.; Touchard, V.; Hemery, P.; Leising, F. Macromolecules 2004, 37, 3214.

4. Yuki, H.; Hatada, K.; Ota, K.; Sasaki, T. Bull. Chem. Soc. Jpn. 1970, 43, 890.

5. Gaussian 03, Revision C.02, Frisch, M. J.; Trucks, G. W.; Schlegel, H. B.; Scuseria, G. E.; Robb, M. A.; Cheeseman, J. R.; Montgomery, J. A.; Vreyen, Jr., T.; Kudin, K. N.; Burant, J. C.; Millam, J. M.; Iyengar, S. S.; Tomasi, J.; Barone, V.; Mennucci, B.; Cossi, M.; Scalmani, G.; Rega, N.; Petersson, G. A.; Nakatsuji, H.; Hada, M.; Ehara, M.; Toyota, K.; Fukuda, R.; Hasegawa, J.; Ishida, M.; Nakajima, T.; Honda, Y.; Kitao, O.; Nakai, H.; Klene, M.; Li, X.; Knox, J. E.; Hratchian, H. P.; Cross, J. B.; Adamo, C.; Jaramillo, J.; Gomperts, R.; Stratmann, R. E.; Yazyev, O.; Austin, A. J.; Cammi, R.; Pomelli, C.; Octhterski, J. W.; Ayala, P. Y.; Morokuma, K.; Voth, G. A.; Salvador, P.; Dannenberg, J. J.; Zakrzewski, V. G.; Dapprich, S.; Daniels, A. D.; Strain, M. C.; Farkas, O.; Malick, D. K.; Rabuck, A. D.; Raghavachari, K.; Foresman, J. B.; Ortiz, J. V.; Cui, Q.; Baboul, A. G.; Clifford, S.; Cioslowski, J.; Stefanov, B. B.; Liu, G.; Liashenko, A.; Piskorz, P.; Komaromi, I.; Martin, R. L.; Fox, D. J.; Keith, T.; Al-Laham, M. A.; Peng, C. Y.; Nanayakkara, A.; Challacombe, M.; Gill, P. M. W.; Johnson, B.; Chen, W.; Wong, M. W.; Gonzalez, C. and Pople, J. A. Gaussian, Inc., Pittsburgh PA, 2004. 